\title{
Non-oscillating solutions to uncoupled Ermakov systems and the semi-classical limit
}

\author{
A Matzkin \\ Department of Physics and Astronomy, University College London, Gower Street, London \\ WC1E 6BT, UK
}

Received 24 January 2001, in final form 7 June 2001

Published 14 September 2001

Online at stacks.iop.org/JPhysA/34/7833

\begin{abstract}
The amplitude-phase formulation of the Schrödinger equation is investigated within the context of uncoupled Ermakov systems, whereby the amplitude function is given by the auxiliary non-linear equation. The classical limit of the amplitude and phase functions is analysed by setting up a semi-classical Ermakov system. In this limit, it is shown that classical quantities, such as the classical probability amplitude and the reduced action, are obtained only when the semi-classical amplitude and the accumulated phase are non-oscillating functions respectively of the space and energy variables. Conversely, among the infinitely many arbitrary exact quantum amplitude and phase functions corresponding to a given wavefunction, only the non-oscillating ones yield classical quantities in the limit $\hbar \rightarrow 0$.
\end{abstract}

PACS numbers: 02.30.Jr, 02.30.-f, 03.65.-w

\section{Introduction}

Systems of the form

$$
\begin{aligned}
\partial_{t}^{2} u(t)+k^{2}(t) u(t) & =\frac{1}{\rho u^{2}(t)} Y(\alpha(t) / u(t)) \\
\partial_{t}^{2} \alpha(t)+k^{2}(t) \alpha(t) & =\frac{1}{u \alpha^{2}(t)} Z(u(t) / \alpha(t))
\end{aligned}
$$

where $Y$ and $Z$ are arbitrary functions of their arguments, are generically known as Ermakov systems. They are characterized by the existence of a first integral, the Ermakov (or LewisRay-Reid) invariant linking the solutions of equations (1) and (2), thereby giving rise to the so-called non-linear superposition principle. Intensive studies of their properties, such as their linearization [1] or their generalization to higher dimensions [2, 3], have been undertaken to extend the remarkable results concerning uncoupled systems, i.e. $Y(\zeta)=0$ and $Z(\zeta)=a^{2} \zeta$ where $a$ is a constant, obtained by Ray and Reid [4, 5]. 
Although the paradigm in physical applications of uncoupled Ermakov systems has been the classical linear time-dependent harmonic oscillator ( $t$ being the time variable), where the full power of Hamiltonian structure [6], Lagrangian mechanics and Noether symmetries [7] have been employed, it has also been remarked that uncoupled systems link the timeindependent linear Schrödinger equation to a non-linear 'auxiliary' equation in the following way:

$$
\begin{aligned}
& \hbar^{2} \partial_{x}^{2} u(x)+p^{2}(x) u(x)=0 \\
& \hbar^{2} \partial_{x}^{2} \alpha(x)+p^{2}(x) \alpha(x)=\frac{\hbar^{2} a^{2}}{\alpha^{3}(x)}
\end{aligned}
$$

where $x$ refers to the space variable, and $p(x, E)$ to its conjugate momentum in classical mechanics. $E$ is the energy, assumed to be conserved, of the system. We have recently shown [8] that the non-linear equation (4) corresponds to the equation for the amplitude function $\alpha(x)$ in the amplitude-phase formulation of the Schrödinger equation, which arises by performing a so-called Milne transform on the wavefunction; the phase function $\phi(x)$ is obtained by integrating the relation $\partial_{x} \phi=\alpha^{-2}$ (see section 2).

We shall be concerned throughout this paper by the oscillatory properties of the solutions of the uncoupled Ermakov system formed by equations (3) and (4) in the specific case of a potential energy function having a single minimum. Actually, since equation (3) defines a Sturm-Liouville problem, the oscillatory properties of the solutions are well-known [9], and we will restrict our analysis to the oscillations, as a function of $x$ and $E$, of the amplitude and phase functions. Our first aim will be to show that absolutely smooth, that is non-oscillating, amplitude-phase functions, can be constructed. Let us recall that the amplitude-phase formulation of the one-dimensional Schrödinger equation is frequently used in quantum scattering theories that explicitly include closed channels, such as quantum defect theory (see [8] and references therein). In these situations, it is of prime importance for $\alpha(x, E)$ to be a smooth function of both the space (usually radial) coordinate $x$ and the energy $E$, since the scattering parameters (e.g. the phase shifts) are defined in terms of amplitude-phase functions. However, by the principle of non-linear superposition (section 2.1), $\alpha$ may be expressed in terms of two independent solutions $u_{1}$ and $u_{2}$ of equation (3)-solutions which as known oscillate between the turning points of the potential. It follows that $\alpha(x)$ generally oscillates between the turning points. In practical implementations of amplitude-phase formalisms, numerical methods aiming at minimizing the amplitude of the oscillations have been devised. We have proposed in [8] such a method based on the invariant and the non-linear superposition principle in the context of Ermakov systems. Here, the main point to be examined consists in the relationship between non-oscillating amplitude-phase functions and the functions obtained in the semi-classical $(\hbar \rightarrow 0$ ) limit. More specifically, we will prove that in this limit, the only non-oscillating solutions are the ones that yield classical quantities: in particular, the only semi-classical phase function that does not oscillate is the classical reduced action, and conversely the quantum continuation, for finite $\hbar$, of the classical reduced action is a non-oscillating function.

To this end, some properties of amplitude-phase functions, their behaviour as a function of $x$ and $E$ and their connection with Ermakov systems will be recalled in section 2. In section 3, we shall discuss semi-classical amplitude-phase functions by setting up a semi-classical Ermakov system; the classical probability amplitude and reduced action will appear as a particular solution of the semi-classical amplitude and phase functions. These results will then be employed in a formal $\hbar$ expansion of the quantum amplitude-phase functions, to prove that provided the first-order functions are non-oscillating, the solutions to each order in $\hbar$ will 
then not oscillate (section 4). This will be followed in section 5 by a discussion of the results, in particular in relation to quantization of classically integrable systems.

\section{Amplitude-phase functions}

Our concern here will be the 'auxiliary' amplitude and phase functions of the Schrödinger equation (3), with $p^{2}(x)=2 m(E-V(x))$, i.e. a particle of mass $m$ and energy $E$ trapped in a potential well $V(x)$ having a single minimum. $V(x)$ is defined on an interval ] $s_{1}, s_{2}$ [ (typically $s_{1,2}= \pm \infty$ or 0 ). Atomic units will be used throughout, except for the $\hbar$ factors which will be re-established where appropriate.

\subsection{Non-linear superposition principle}

We discuss in this section the main results concerning amplitude-phase functions that will be useful in what follows, omitting details (for more details and the relevant references, see [8]). Equation (3) defines a Sturm-Liouville problem, typically a singular problem on the half line or real line when vanishing boundary conditions at $s_{1}$ and $s_{2}$ are implemented. However, our interest lies not in the specific eigenfunctions or eigenvalues of the Schrödinger equation, but in relating linearly independent solutions of the linear equation to amplitude and phase functions. We will denote by $u_{1}$ and $u_{2}$ two independent solutions of equation (3), respectively, regular at $s_{1}$ and $s_{2}$ and with Wronskian $W=\mathcal{W}\left[u_{1}, u_{2}\right] \equiv\left(\partial_{x} u_{1}\right) u_{2}-u_{1}\left(\partial_{x} u_{2}\right)$.

A general solution $u(x)$ of equation (3), which is readily written in terms of the independent solutions $u_{1}$ and $u_{2}$, may also be obtained as

$$
u(x)=b_{1} \alpha(x) \sin \left[\phi(x)+b_{2}\right]
$$

where $b_{1}$ and $b_{2}$ are complex constants. A straightforward substitution in equation (3) leads to the two equations

$$
\begin{aligned}
& \partial_{x}^{2} \alpha(x)+p^{2}(x) \alpha(x)=\alpha(x)\left[\partial_{x} \phi(x)\right]^{2} \\
& \alpha^{2}(x)=\frac{a}{\partial_{x} \phi}
\end{aligned}
$$

where we can set $a^{2}=1$ without any loss of generality (since $a^{2}$ can be absorbed into $\alpha$ by redefining $\alpha \rightarrow \alpha / a^{1 / 2}$ ), thus recovering equation (4). For obvious reasons, $\alpha$ and $\phi$ are known respectively as the amplitude and phase functions. In terms of $u_{1}$ and $u_{2}$, it follows from standard results on Ermakov systems that the general solution for $\alpha$ is given by

$$
\alpha(x)=\left[\left(\frac{1}{2 I}+2 I c^{2}\right) u_{1}^{2}(x)+\frac{2 I}{W^{2}} u_{2}^{2}(x)-\frac{4 I c}{W} u_{1}(x) u_{2}(x)\right]^{1 / 2}
$$

and the equation for the phase is readily integrated to give

$$
\phi(x)=\arctan \left[\left(\frac{1}{2 I}+2 I c^{2}\right) W \frac{u_{1}(x)}{u_{2}(x)}-2 I c\right]+\arctan 2 I c
$$

where the integration constant is chosen so that $\phi\left(s_{1}\right)=0$. Equation (8) is an illustration of the non-linear superposition principle [7]. $I$ and $c$ are two constants, independent of $x$ ( $I$ is the Ermakov, or Lewis-Ray-Reid, invariant). Note that the value of the phase function at $s_{2}$, known as the accumulated phase, does not depend on the constants $I$ and $c$ for the eigenvalues $E_{0}$ of the Sturm-Liouville problem, since

$$
\begin{aligned}
& \phi\left(s_{2}, E=E_{0}\right)=\pi n \\
& \phi\left(s_{2}, E \neq E_{0}\right)=\arctan [2 I c(E)]+\frac{2 n+1}{2} \pi .
\end{aligned}
$$


Here $n$ is an integer giving the number of nodes of $u_{2}$. Note also that $\alpha\left(x_{1}\right)$ is independent of $c$ if $x_{1}$ is a zero of $u_{1}$.

\subsection{Boundary conditions and energy dependence}

The boundary conditions for $\alpha$ and $\phi$ are therefore incorporated through the parameters $I(E)$ and $c(E)$, which as indicated depend on the energy. Normalization of the eigenfunctions $f$ of the Sturm-Liouville problem with vanishing boundary conditions at $s_{1}$ and $s_{2}$, yields

$$
\int_{s_{1}}^{s_{2}} f^{2}(x) \mathrm{d} x=I \partial_{E} \phi\left(s_{2}, E=E_{0}\right) .
$$

By choosing the eigenfunctions to be normalized per unit energy increment, $I$ becomes an energy-independent positive constant, and amplitude and phase functions depend on the single parameter $c(E)$.

We now introduce another solution $g(x)$ of the Schrödinger equation (3), defined in terms of the solutions regular at $s_{1}$ and $s_{2}$ as

$$
g(x, c)=2 I\left(\frac{u_{2}(x)}{W}-c u_{1}(x)\right)
$$

which fulfils $\mathcal{W}\left[u_{1}, g\right]=2 I$ and gives $\alpha(x)=\left[\frac{1}{2 I}\left(u_{1}^{2}(x)+g^{2}(x)\right)\right]^{1 / 2}$. This simply means that $u_{1}$ and $g$ lag $\pi / 2$ out of phase, and that with the conventions of section $2.1(a=1$ and $\left.\phi\left(s_{1}\right)=0\right)$, we have

$$
\begin{aligned}
& u_{1}(x)=\sqrt{2 I} \alpha(x, c) \sin \phi(x, c) \\
& g(x, c)=\sqrt{2 I} \alpha(x, c) \cos \phi(x, c) .
\end{aligned}
$$

We have emphasized the $c$-dependence of the different functions (though self-consistency requires it, it may be checked explicitly that $u_{1}$ does not depend on $c$ ).

\subsection{Oscillatory properties}

2.3.1. Oscillations of the amplitude. Let $t_{1}(E)\left(t_{2}(E)\right)$ be the inner (outer) turning point. For values beyond the turning points (when $x<t_{1}$ or $x>t_{2}$ ), we recast equation (6) as

$$
\frac{1}{2}\langle\phi ; x\rangle=p^{2}(x)-\alpha^{-4}(x)
$$

where $\langle\phi ; x\rangle \equiv \partial_{x}^{3} \phi / \partial_{x} \phi-\frac{3}{2}\left(\partial_{x}^{2} \phi / \partial_{x} \phi\right)^{2}$ denotes the Schwartzian derivative. Since $\alpha$ is a positively defined quadratic form and $p^{2}(x)<0$ beyond the turning points, the Schwartzian derivative of the phase is negative, and as it can be verified, if $\langle\phi ; x\rangle<0$ on an interval then $\partial_{x} \phi$ cannot have a positive local minimum on this interval, i.e. $\alpha$ cannot have a local maximum. By noting that $\alpha(x) \rightarrow+\infty$ when $x \rightarrow s_{1}$ and $x \rightarrow s_{2}$, we conclude that if $\alpha$ does not oscillate between the turning points, it will not oscillate on the whole interval $] s_{1}, s_{2}[$. Note that there is then a unique value $x_{0}$, with $t_{1}<x_{0}<t_{2}$, such that $\partial_{x} \alpha\left(x_{0}\right)=0$.

However, as $u_{1}(x)$ and $u_{2}(x)$ oscillate for $t_{1}<x<t_{2}, \alpha$ will generally oscillate, by virtue of the non-linear superposition principle equation (8), between the turning points, the local wavelength being half that of $u_{1}$ or $u_{2}$. Nonetheless, at a specified energy, there may be infinitely many values of $c$ giving a non-oscillating amplitude function ${ }^{1}$. We give in the

1 For example, taking the second derivative of $\alpha$, it is seen that any value of $c$ enclosed between $c_{ \pm}(x)$, with $c_{ \pm}(x)=u_{2}(x)\left[W u_{1}(x)\right]^{-1} \pm\left[2 I u_{1}(x)\right]^{-1}\left[2 I / p(x)-u_{1}^{2}(x)\right]^{1 / 2}$ and where $x$ spans the interval between the turning points, will suffice. 
following sections a sufficient (but not necessary) condition for $c(E)$, that is the value of $c$ and its explicit energy dependence, because this value has remarkable properties related to classical quantities in the semi-classical limit, as will be seen in section 3.

2.3.2. Inverted phase accumulation. Proceeding as in section 2.1, we can define a phase function $\bar{\phi}(x)$ such that $\bar{\phi}\left(s_{2}\right)=0$, i.e. the phase starts accumulating at $s_{2}$ instead of $s_{1}$. As can be easily seen, this amounts to an exchange of the roles of $u_{1}$ and $u_{2}$; for instance, we now have $u_{2}=b_{2} \bar{\alpha} \sin \bar{\phi}$, where $\partial_{x} \bar{\phi}=\bar{\alpha}(x)^{-2}$ and $b_{2}$ is a constant to be set below. $\bar{\alpha}$ is given by

$$
\bar{\alpha}^{2}(x)=\left(\frac{1}{2 I}+2 I \bar{c}^{2}\right) u_{2}^{2}(x)+\frac{2 I}{W^{2}} u_{1}^{2}(x)+\frac{4 I \bar{c}}{W} u_{1}(x) u_{2}(x)
$$

with $\mathcal{W}\left[u_{2}, u_{1}\right]=-W$ and where we assumed $I=\bar{I}$ for simplicity. Generally $\alpha(x, c)$ and $\bar{\alpha}(x, \bar{c})$ are very different functions (e.g. the $\bar{c}$ independent points of $\bar{\alpha}$ are now located at the zeros of $\left.u_{2}\right)$. Notwithstanding, it is apparent that $\alpha(x, c)=\bar{\alpha}(x, \bar{c})$ iff $c^{2}=\bar{c}^{2}=W^{-2}-(2 I)^{-2}$ and $\bar{c}=-c$. We shall set

$$
c_{0}(E)=-\left[[W(E)]^{-2}-[2 I]^{-2}\right]^{1 / 2} .
$$

To keep the quadratic form real we shall set $W^{2}<4 I^{2}$, a condition to be assumed in the rest of the paper (this is not a problem in practice because $\alpha$ and $\phi$ are left unchanged by the transformations $u_{1} \rightarrow \kappa u_{1}, W \rightarrow \kappa W, I \rightarrow \kappa^{2} I, c \rightarrow c / \kappa^{2}$, so the Wronskian can be conveniently rescaled).

Let us now suppose that $c=-\bar{c}= \pm c_{0}$. We then have $\alpha=\bar{\alpha} . b_{2}$ is found by evaluating $\mathcal{W}\left[u_{1}, u_{2}\right]$ at $s_{2}$, which yields, by choosing a proper sign convention, $b_{2}=\sqrt{2 I}$. From equations (13)-(15), it follows that

$$
\sin \bar{\phi}\left(\mp c_{0}\right)=W\left[\cos \phi\left( \pm c_{0}\right) / 2 I \mp\left[W^{-2}-(2 I)^{-2}\right]^{1 / 2} \sin \phi\left( \pm c_{0}\right)\right]
$$

thereby obtaining the relation between $\phi$ and $\bar{\phi}$, which gives the oscillations of $u_{1}$ and $u_{2}$ only if $\alpha$ does not oscillate.

2.3.3. Auxiliary quadratic form on the unit circle. $\alpha^{2}(x)$ is a positive definite quadratic form. Labelling $M$ the matrix of the coefficients, we have $\operatorname{det} M=W^{-2}$ and $\operatorname{Tr} M=$ $1 / 2 I+2 I c^{2}+2 I / W^{2} \cdot \alpha^{2}(x)$ can be reduced to the canonical form

$$
\alpha^{2}(x, c)=\lambda_{1}(c) v_{1}^{2}(x, c)+\lambda_{2}(c) v_{2}^{2}(x, c)
$$

where $\lambda_{i}(c)$ are the eigenvalues of $M\left(\lambda_{1} \geqslant \lambda_{2}\right)$ and $v_{i}(x, c)$ are the eigenvectors, normalized so that $v_{1}^{2}(x, c)+v_{2}^{2}(x, c)=u_{1}^{2}(x)+u_{2}^{2}(x)$. We now introduce a quadratic form $Q$ defined as

$$
Q(x, c)=\lambda_{1}(c) w_{1}^{2}(x, c)+\lambda_{2}(c) w_{2}^{2}(x, c)
$$

where $w_{i}^{2}(x, c)=v_{i}^{2}(x, c)\left[u_{1}^{2}(x)+u_{2}^{2}(x)\right]^{-1}$. As indicated, $Q$, as well as the $\lambda_{i}$ and $v_{i}$ are $c$-dependent. $Q$ oscillates between its maximum and minimum values, which are by construction given respectively by $\lambda_{1}$ and $\lambda_{2}$.

We now set $c= \pm c_{0}$. Let $x_{1}\left(x_{2}\right)$ label the points where $u_{1}\left(u_{2}\right)$ vanishes; we then have $Q\left(x_{1,2}, \pm c_{0}\right)=2 I / W^{2}$, so that between two zeros of $u_{1}$ and $u_{2}, Q\left(x, \pm c_{0}\right)$ has at least one extremum on the unit circle (for these points, the equality $u_{1}^{2}(x)=u_{2}^{2}(x)$ is fulfilled). Note that $\alpha\left(x, c= \pm c_{0}\right)$ goes through both the $c$-independent points of $\alpha(x, c)$ and $\bar{\alpha}(x, \bar{c})$, so if $\alpha$ oscillates, then there is an extremum of $\alpha$ between $x_{1}$ and $x_{2}$, and the sign of $\partial_{x} \alpha$ alternates between the consecutive zeros of $u_{1}$ and $u_{2}$. This is illustrated in figure 1 for the specific case of the harmonic oscillator (to be discussed in details in section 5.4); the zeros of $u_{1}$ and $u_{2}$ are respectively shown as triangles and rectangles. Note also that the maxima of $Q\left(c_{0}\right)$ 


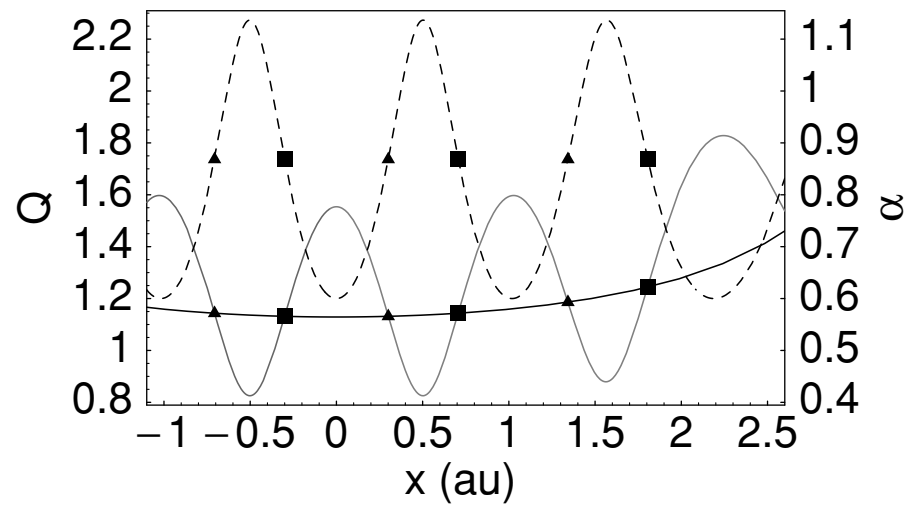

Figure 1. $Q\left(c_{0}\right)$ (broken line, left scale), $\alpha\left(c_{0}\right)$ (non-oscillating solid line, right scale) and $\alpha\left(-c_{0}\right)$ (oscillating solid line) are plotted for a harmonic oscillator (atomic units and $\omega=1, n(E)=4.4$, see section 5.4 for the definitions). All amplitude functions $\alpha(c)$ for any $c$ go through the points $x_{1}$ (triangles), and any function $\bar{\alpha}(\bar{c})$ goes through the points $x_{2}$ (rectangles). Only the two functions $\alpha\left( \pm c_{0}\right)$ go through both the points $x_{1}$ and $x_{2}$. Since $Q$ oscillates and $Q\left(x=x_{1,2}, \pm c_{0}\right)=2 I / W^{2}$ is constant, $\partial_{x} Q$ has opposite signs at $x_{1}$ and $x_{2}$ as may be seen in the figure. This is also the case for $\alpha\left(-c_{0}\right)$ (solid line) which therefore oscillates, but not for $\alpha\left(c_{0}\right)$.

correspond to the minima of $Q\left(-c_{0}\right)$, since $Q\left(-c_{0}\right)=\bar{Q}\left(c_{0}\right)$. Combining equations (20) and (21) and taking the derivative $\partial_{x} \alpha\left(x_{1,2}\right)$ as a function of $\partial_{x} Q, Q, u_{1}$ and $u_{2}$, it can be seen indeed that for $c=-c_{0}$ the sign of $\partial_{x} \alpha$ at two consecutive zeros, $x_{1}$ and $x_{2}$, alternates. However, for $c=c_{0}$, the sign of $\partial_{x} \alpha$ between two consecutive arbitrary zeros of $u_{1}$ and $u_{2}$ does not change, and thus $\alpha\left(c_{0}\right)$ does not oscillate ${ }^{2}$.

2.3.4. Oscillations of the accumulated phase. We have explained in [8] why obtaining nonoscillatory functions is important when amplitude-phase methods are employed in scattering theory. The goal was to extend energy normalization, which for the eigenfunctions of the Sturm-Liouville problem is given by equation (12), to functions $f(x)$ which converge at $s_{2}$ but diverge at $s_{1}$ (the phase-shifted or scattered wavefunctions). By combining the continuity equation for the probability density and L'Hôpital's rule, improper energy normalization is defined as

$$
\int_{r}^{s_{2}} f^{2}(x, E) \mathrm{d} x=I \partial_{E} c\left(\frac{1}{2 I}+2 I c^{2}(E)\right)^{-1}
$$

where $r$ is a cut-off radius (and as above, $I$ is assumed to be energy-independent). This normalization is of course arbitrary, since it is governed by $c(E)$, but it conditions the energy dependence of the different scattering parameters. In particular, the accumulated phase, which is unambiguously defined (equation (10)) for the eigenfunctions of the Sturm-Liouville problem, crucially depends (equation (11)) on the normalization when $E$ is not an eigenvalue.

More precisely, let us assume the eigenvalues of the Sturm-Liouville problem to be given by $E_{0}=\xi(n)$, where $n$ is the number of zeros of the corresponding eigenfunction (thus,

\footnotetext{
2 We noted, however, that given the behaviour of the amplitude function at $s_{1}$ and $s_{2}$, there is necessarily a point between $t_{1}$ and $t_{2}$ where $\partial_{x} \alpha$ vanishes. The argument sketched here relies on the signs of the basis functions $u_{1}$ and $u_{2}$ and their derivatives when $V(x)$ is monotonous on a full cycle of oscillation of the basis functions, and excludes the neighbourhood around the bottom of the potential, where $\partial_{x} \alpha$ changes sign (but $\partial_{x} \alpha$ does not vanish exactly at the bottom of the potential, as would be the case in the WKB approximation).
} 
of $\left.u_{2}\right)$, and $\xi(n)$ is an a priori arbitrary, but monotonous function admitting a differentiable inverse, $n(E)=\xi^{-1}(E)$. The functional relation between $E_{0}$ and integer values of $n$ is thereby extended to any energy $E$ lying between two eigenvalues, i.e. $E=\xi(n), n$ real. The energy dependence for $c(E)$ can now be chosen so as to extend the normalization of the eigenfunctions to non-integer values of $n$ by equating equation (22) to $I \pi \partial_{E} \xi^{-1}(E)$ (cf. equations (10) and (12)), yielding

$$
c(E)=-\frac{1}{2 I} \cot \pi \xi^{-1}(E) .
$$

Substituting in equation (11) gives the following expression for the accumulated phase:

$$
\phi\left(s_{2}, E \neq E_{0}\right)=\pi \xi^{-1}(E) \equiv \pi n(E) .
$$

Thus the accumulated phase does not oscillate as a function of the energy (it is a simple straight line as a function of $n$ ) and the value of $c$ given in equation (23) is the only value compatible with energy normalization leading to a non-oscillating accumulated phase function. We shall mention in section 5.3 the relation the specific form (24) has with the canonical action variable in classical mechanics. Note finally that, provided the basis functions $u_{1}$ and $u_{2}$ are redefined so that their Wronskian is proportional to $2 I \sin \pi n(E)$, equation (23) becomes a particular form of the more general equation (18): with such a choice, the amplitude $\alpha$ is a non-oscillating function of $x$ and the accumulated phase $\phi\left(s_{2}\right)$ is a non-oscillating function of $E$.

\section{Semi-classical Ermakov system}

\subsection{Asymptotic solutions to the linear equation}

The approximate solutions to the one-dimensional Schrödinger equation when $\hbar \rightarrow 0$ are well known from the asymptotic theory of ordinary linear differential equations [10]. It follows from section 2.3.1 that it is sufficient to consider the solutions between the turning points (i.e. for real $p(x))$. Real solutions are of the form

$$
\tilde{u}(x)=\frac{a_{1}}{\sqrt{p(x)}} \sin \left[ \pm \int p\left(x^{\prime}\right) \mathrm{d} x^{\prime}+a_{2}\right]
$$

where $a_{1}$ and $a_{2}$ are constants. Tilded $\left(^{\sim}\right)$ quantities will henceforth denote asymptotic (semiclassical) functions when these are to be distinguished from the corresponding exact quantum solutions. It is well known from the Hamilton-Jacobi theory that

$$
S(x, E)= \pm \int p\left(x^{\prime}, E\right) \mathrm{d} x^{\prime}+a_{2}
$$

where $S(x, E)$ is known as the Hamilton-Jacobi characteristic function or reduced action: the characteristics in phase space are made up of the points $\left(x, \partial_{x} S\right)$.

\subsection{Ermakov system}

By direct substitution of a general asymptotic solution into the Schrödinger equation, and by labelling $\tilde{\alpha}$ and $\tilde{\phi}$ the semi-classical amplitude and phase functions, we obtain a semi-classical Ermakov system

$$
\begin{aligned}
& \hbar^{2} \partial_{x}^{2} \tilde{u}+\left[p^{2}(x)+\frac{\hbar^{2}}{2}\langle S ; x\rangle\right] \tilde{u}=0 \\
& \hbar^{2} \frac{\partial_{x}^{2} \tilde{\alpha}}{\tilde{\alpha}}+\left[p^{2}(x)+\frac{\hbar^{2}}{2}\langle S ; x\rangle\right]=\hbar^{2}\left(\partial_{x} \tilde{\phi}\right)^{2}
\end{aligned}
$$


where again the bracket $\langle; x\rangle$ denotes a Schwartzian derivative and we have, as for the usual amplitude-phase functions, $\tilde{\alpha}^{2}=\tilde{a} / \partial_{x} \tilde{\phi}$ and thus $\partial_{x}^{2} \tilde{\alpha} / \tilde{\alpha}=-\langle\tilde{\phi} ; x\rangle / 2$. Equations (27) and (28) are the semi-classical version of the quantum system given by equations (3) and (4). Equation (27) is the modified Schrödinger equation exactly obeyed by the semi-classical wavefunctions, and equation (28) is the non-linear equation fulfilled by the semi-classical amplitude function. The passage from the exact (quantum) Ermakov system to the semiclassical one simply consists in a redefinition of the potential energy function, which is identical if $\langle S ; x\rangle$ vanishes. Although generally $\langle S ; x\rangle$ is non-zero (except for the free particle), $\langle S ; x\rangle$ does tend to zero or to a finite value in the limit of high quantum numbers (e.g. the harmonic oscillator for the former, the centrifugal Coulomb potential for the latter). Only if this value is negligible compared to the other terms in the energy function does the high quantum numbers condition fit with the semi-classical limit.

\subsection{General solutions}

$\tilde{\alpha}$ and $\tilde{\phi}$ are given in terms of two independent functions $\tilde{u}_{1}$ and $\tilde{u}_{2}$ of equation (27) by the same relations, equations (8) and (9), as in the exact (quantum) case, with now tilded quantities. It is convenient, however, to set the tilded constants $\tilde{a}, \tilde{I}$ and $\tilde{W}$ equal to their quantum counterpart $a, I$ and $W$. This is done by first noting that $\mathcal{W}[\alpha \sin \phi, \alpha \cos \phi]=a$, which we then set equal to $\mathcal{W}[\tilde{\alpha} \sin \tilde{\phi}, \tilde{\alpha} \cos \tilde{\phi}]$, so $\tilde{a}=a=1$. To preserve the Wronskians (cf. equations (14) and (15)), the semi-classical function $\tilde{u}_{1}$, of the form given by equation (25), is thus set as

$$
\tilde{u}_{1}(x)=\sqrt{\frac{2 I}{p(x)}} \sin S(x)
$$

where we have implicitly included $a_{2}$ in the reduced action so that $\tilde{u}_{1}$ is the asymptotic approximation to $u_{1}$ in the neighbourhood of an arbitrary $x$ lying between the turning points. An independent solution $\tilde{u}_{2}$ with Wronskian $\mathcal{W}\left[\tilde{u}_{1}, \tilde{u}_{2}\right]=W$ is then obtained under the form $\cos (S(x)+b) / \sqrt{p(x)}$ as

$$
\tilde{u}_{2}(x)=\sqrt{\frac{2 I}{p(x)}} \kappa \cos \left[S(x)+\arccos \frac{W}{2 I \kappa}\right]
$$

where we have introduced the scaling factor $\kappa$ to keep all quantities real. In what follows, we shall set $\kappa=1$, which is tantamount to rescaling $u_{2}$ (a similar rescaling was performed in the quantum case, see below equation (18)).

\subsection{Non-oscillating solutions}

Substituting equations (29) and (30) in the expression for $\tilde{\alpha}$ readily yields

$$
\begin{aligned}
\tilde{\alpha}^{2}=\frac{1}{p(x)}[ & I^{2} W^{-2} \sin ^{2} S(x)+(\cos S(x)-2 I c \sin S(x)) \\
& \left.\times\left\{\cos S(x)-2 I\left(c+W^{-1}\left[4-W^{2} / I^{2}\right]^{1 / 2}\right) \sin S(x)\right\}\right]
\end{aligned}
$$

which is a highly oscillatory function for an arbitrary value of the parameter $c$. However, it may be noted by inspection that for $c=-\left[W^{-2}-(2 I)^{-2}\right]^{1 / 2}$, the oscillating terms are cancelled out. Remark that this is the same expression that was labelled $c_{0}$ in the quantum case (equation (18)). Re-establishing $\hbar$, the amplitude now reads $\tilde{\alpha}^{2}\left(c_{0}\right)=h / p(x)$, which given our assumptions is a non-oscillating function of $x$. Identical substitutions may be done 
for $\tilde{\phi}$, from which it follows that the semi-classical phase function is highly oscillatory for an arbitrary value of $c$ except if $c=c_{0}$, and in that case,

$$
\tilde{\phi}\left(x, c_{0}\right)=S(x) / \hbar \text {. }
$$

In short, non-oscillating functions are obtained for a unique value of the parameter $c$, for which the semi-classical quantities match their classical counterpart $(\sqrt{\hbar / p(x)}$ and $S(x) / \hbar$ are respectively the classical probability amplitude and phase functions). Writing equation (28) as

$$
\frac{\hbar^{2}}{2}[\langle\tilde{\phi} ; x\rangle-\langle S ; x\rangle]=p^{2}(x)-\frac{\hbar^{2}}{\tilde{\alpha}^{4}}
$$

means that each side of the non-linear equation of the semi-classical Ermakov system vanishes independently. Note also that $p^{2}(x)+\hbar^{2}\langle S ; x\rangle / 2=\hbar^{2}\langle\tan \tilde{\phi}(c) ; x\rangle / 2$ (this is established by using the Möbius invariance of the Schwartzian derivative and establishing a $c$-dependent linear transformation relating $\tan \tilde{\phi}(x, c)$ to $\tan S(x))$.

\section{4. $\hbar$ expansions}

The link between the solutions of the quantum and semi-classical Ermakov systems is done by employing a formal $\hbar$ expansion of the amplitude function. As in the previous section, we assume $I$ and $W$ to be identical in both the quantum and the semi-classical case. It is then straightforward to show that to each order in $\hbar$ (as well as to infinite order), non-oscillatory functions are obtained.

\subsection{Series expansions}

The formal asymptotic solution to the Schrödinger equation for small values of the parameter $\hbar$ is usually done by transforming it to the Riccati form and then obtaining a recurrence relation between complex function of order $j$ and the functions of lower order [10]. Here we proceed slightly differently, because we want the relations between the amplitude and the phase to be verified to each order. We look for a generic solution of equation (3) under the form $u(x)=a(x)$ exp if $(x) / \hbar$, where $a(x)$ and $f(x)$ are real functions admitting the series expansions

$$
a(x)=\sum_{j=0}^{\infty} a_{j}(x) \hbar^{j} \quad f(x)=\sum_{j=0}^{\infty} f_{j}(x) \hbar^{j} .
$$

Substitution into the Schrödinger equation gives $a_{j}(x)=0, f_{j}(x)=0$ for odd $j$ and the following recurrence relations for even $j, j \geqslant 2$ :

$$
\begin{aligned}
& \partial_{x} f_{j}(x)=\frac{1}{2 a_{0} \partial_{x} f_{0}}\left[\partial_{x}^{2} a_{j-2}-\sum_{m=2}^{j-2} a_{m} \sum_{n=0}^{j-m} \partial_{x} f_{n} \partial_{x} f_{j-m-n}\right] \\
& a_{j}(x)=\frac{b_{j}}{\sqrt{\partial_{x} f_{0}}}-\sum_{n=0}^{j-2} \frac{\int\left(2 \partial_{y} a_{n} \partial_{y} f_{j-n}+a_{n} \partial_{y}^{2} f_{j-n}\right) / \sqrt{\partial_{y} f_{0}} \mathrm{~d} y}{2 \sqrt{\partial_{x} f_{0}}}
\end{aligned}
$$

with $f_{0}(x)= \pm S(x), a_{0}(x)= \pm \partial_{x} f_{0}^{-1 / 2}$, and where the constants $b_{j}$ appearing in the solution to the homogeneous equations for $a_{j}(x)$ are all set to 0 for $j \geqslant 2$. The Wronskian relations are then preserved to each order in $\hbar$, that is

$\mathcal{W}[a(x) \sin f(x), a(x) \cos f(x)]=a_{0}^{2}(x) \partial_{x} f_{0}(x)=\partial_{x} S(x) / p(x)=1$. 
Between the turning points, the \pm branches are combined to yield real oscillatory functions. The formal expansion for $u_{1}$ (assuming again the adequate integration constant to be included in $f_{0}$ ) is then given by

$$
u_{1}(x)=\sqrt{2 I} \sum_{j=0}^{\infty} a_{j}(x) \hbar^{j} \sin \left(\sum_{i=0}^{\infty} f_{i}(x) \hbar^{i}\right) .
$$

The expansion for the function lagging $\pi / 2$ out of phase is trivially obtained by using the cos function; from equations (13) to (15) the formal expansion for $u_{2}$ is then found as

$u_{2}(x)=\frac{\sum_{j=0}^{\infty} a_{j}(x) \hbar^{j}}{\sqrt{2 I}}\left[W \cos \left(\sum_{i=0}^{\infty} f_{i}(x) \hbar^{i}\right)-I\left[4-W^{2} / I^{2}\right]^{1 / 2} \sin \left(\sum_{i=0}^{\infty} f_{i}(x) \hbar^{i}\right)\right]$.

To first order these functions coincide by construction with the semi-classical wavefunctions $\tilde{u}_{1}$ and $\tilde{u}_{2}$.

\subsection{Amplitude and phase expansions}

The $\hbar$ expansions for the amplitude and phase functions are obtained by combining the nonlinear superposition principle [equations (8) and (9)] with the formal series expansions for $u_{1}$ and $u_{2}$. The expansions may be done to finite or infinite order. In the first case, the functions are Taylor expanded around $\hbar=0$ after separating the classical terms $a_{0}$ and $f_{0}$. The infiniteorder case is analogous to the first-order case treated in section 3.4. For example, substituting the series expansions in equation (9) gives the expression $(\bmod \pi)$ of the phase which can be simplified as

$\phi(x, c)=\operatorname{arccot}\left\{\cot \left(\frac{S(x)}{\hbar}+\sum_{i=1}^{\infty} f_{i}(x) \hbar^{i-1}\right)-\left[2 I c(E)+I W^{-1}\left[4-W^{2} / I^{2}\right]^{1 / 2}\right]\right\}$.

The amplitude function may be obtained by deriving equation (40), keeping in mind the Wronskian relations (37), yielding an expression involving the sines and cosines of the expression between parentheses. In both cases, the highly oscillatory terms are cancelled by setting $c=c_{0}$ (in equation (40) for example, the term between square brackets then vanishes).

\section{Discussion}

\subsection{General remarks}

We have thus seen that same value of $c$ gives rise to non-oscillating functions both in the semi-classical and quantum cases. This is not surprising, if the similarities between equations (30), (39) and (19) on the one hand, together with the non-linear superposition principle in both the quantum and semi-classical Ermakov systems on the other hand are considered: the same functional relation gives the amplitude-phase functions in both cases. We note however that the simple arguments we have given above, although physically appealing because of the direct connection to standard classical quantities, are liable to a more rigorous treatment. For example the series expansion obtained for the amplitude function does not necessarily converge, and even its asymptotic properties as a function of the parameter $c$ deserve a more thorough investigation. Actually, Lewis had made the same remarks when studying the 
adiabatic invariant series of the time-dependent classical harmonic oscillator in powers of an adiabatic parameter $\varepsilon$ [11], which is defined by the same uncoupled Ermakov system as the one studied in this paper. Lewis's work was the first (albeit implicit) application to Ermakov systems of Kruskal's asymptotic theory of Hamiltonian systems extending the study of the adiabatic invariants beyond the first order in $\varepsilon$ [12]. The transposition of these theories to the present problem is not straightforward because we lack here the Hamiltonian formalism on which these theories are based (e.g. the integral invariants that appear in Kruskal's theory would have here a rather obscure interpretation). Nonetheless, the present results on the oscillatory properties can be directly transposed to any uncoupled Ermakov system depending on an 'adiabatic' parameter $\varepsilon$ (which in the present context corresponds of course to the Planck constant).

\subsection{Scattering basis functions}

Previously to the work of Fano et al [13], the use of amplitude-phase methods in scattering theory was limited to the high kinetic energy limit, and the derivatives of the phase $\phi$ above first order neglected, thereby effectively restricting the treatment from the start to the standard WKB approximation (see e.g. chapter 4.3 of [14]). The more recent application of these methods to define a pair of basis functions for phase-shifted wavefunctions in a potential (as outlined above) relies on numerical treatments to minimize the oscillations; these treatments are preferred even in the cases for which an analytic pair of basis functions is known, such as the Whittaker functions for the centrifugal Coulomb problem or the parabolic cylinder functions for the harmonic oscillator. It is interesting to note that the approach suggested in section 2.3 yields, for an arbitrary potential with a single minimum, the same relations that are known to be valid (and non-oscillating) for the analytic functions in the mentioned special cases (e.g. in the Coulomb case where the effective quantum number $v$ is defined as $v=\xi^{-1}(E)+l$, the accumulated phase obtained with the Whittaker functions is $\phi(\infty)=\pi(v-l)$ and improper normalization follows the normalization of the eigenfunctions by normalizing to $v^{3} / 2$, independently of $v$ being real or an integer [15]).

From a formal standpoint, defining a specific basis of functions is equivalent to defining the Green's function of the scattering process in the asymptotic field. This is the Green's function that appears in the Lippmann-Schwinger equations and through which the collision operators are defined. This is why the collision operators depend on the parameter $c$. Though at first sight this may appear as an unexpected feature, it must be remembered that the explicit inclusion of closed channels leads to a modification of the usual Green's functions through a term depending on the accumulated phase [16].

\subsection{Semi-classical limit and classical quantities}

Standard semi-classical physics is usually not concerned with the quantum to classical limiting procedure, because the strategy there is to start from classical quantities at the outset and then proceed to quantization. However, we have seen in section 3 that the classical reduced action is only one of the many phase functions that are obtained in the semi-classical limit (namely the non-oscillating one). This type of problem is frequent in 'classicalization' procedures: a particular, often arbitrary choice has to be made to recover classical quantities (action, Liouville equation, etc.). A recent example is given in reference [17], where the passage from Hilbert-space to classical-phase space operators involves particular choices for the parameters in order to recover the classical dynamics. In other works, this requirement takes the form of an additional ad hoc condition usually termed as the 'correspondence principle' (e.g. in 
reference [18] where a quantum version of the Hamilton-Jacobi equation is given an additional boundary condition $p_{\text {quantum }}(x, E) \rightarrow p_{\text {classical }}(x, E)$ when $\hbar \rightarrow 0$ and $E$ is fixed $)^{3}$. Though much less general, our treatment is more transparent in that the continuation of the classical reduced action in the quantum domain is readily identified: it is the non-oscillating phase function (and the continuation of the classical probability amplitude is the non-oscillating amplitude function).

Other aspects of the classical-quantum correspondence for a classically integrable and separable system deserve to be mentioned. The accumulated phase (equations (10) and (11)) is seen to be directly related to the line integral around a closed loop of $\alpha^{-2}$ :

$$
\oint\left[\partial_{x} \phi(x, c)\right] \mathrm{d} x=2 \phi\left(s_{2}, c\right) .
$$

In contrast to EBK (torus) quantization, there are no caustics when dealing with exact quantization, and the quantization condition reads $\oint\left[\partial_{x} \phi(x, c)\right] \mathrm{d} x=2 \pi n$, i.e., $n$ is an integer and the line integral does not depend on the particular value of $c$. Here we understand by 'exact quantization' the quantization of the exact quantum phase, and not the exact WKB quantization of non-solvable potentials, as employed by Voros [19]. Note however that when $c=c_{0}$, the (unquantized) integral (41) reads, according to equation (24), as

$$
\oint\left[\partial_{x} \phi\left(x, c_{0}\right)\right] \mathrm{d} x=2 \pi n(E) .
$$

Not only does $c=c_{0}$ preserve for non-integer real numbers the functional relation valid for exact quantization, but it gives a parameterization of the quantum equivalent of the canonical action variable.

In a similar vein, the period of motion $T$ is given in the Hamilton-Jacobi theory by taking the energy derivative of the reduced action along the closed loop. The transposition to the phase function in the quantum case would imply taking the energy derivative of equation (41), which by equations (11) and (22) is proportional to the normalization. Again, the normalization depends on $c(E)$ and to take $\partial_{E} \phi(c)$ as the time parameterization does not appear to make much sense unless $c=c_{0}$, since any other value would lead to an oscillating function, which would further not collapse to the classical period in the $\hbar \rightarrow 0$ limit.

\subsection{Example}

We illustrate the properties mentioned above on the harmonic oscillator, a paradigm both in the Hamilton-Jacobi formulation of classical mechanics [20] and in the semi-classical theory of bound states [21]. The reduced action $S(x, E)$ of equation (26) is readily obtained, from which it follows that

$$
\begin{gathered}
\partial_{x} S=\left(2 m E-m^{2} \omega^{2} x^{2}\right)^{1 / 2} \\
J \equiv \oint\left[\partial_{x} S\right] \mathrm{d} x=2 \pi E / \omega
\end{gathered}
$$

where $m$ and $\omega$ are the mass and frequency of the oscillator respectively; we slightly depart from usual conventions and define $J$ to be the canonical action variable. The period is recovered as $T=\partial_{E} J$. The standard WKB solutions between the caustics, equations (29) and (30), are

3 Other authors crudely suppress the $\hbar$-dependent terms in selected equations where this suppression leads to classical relations (e.g. this would be done in equation (4) by giving the amplitude squared the dimensions of a classical quantity, thereby getting rid of $\partial_{x}^{2} \alpha$, but would not be done in equation (3), which does not support an obvious classical interpretation). This procedure has often been criticized because the functions and the parameters appearing in the equations depend on $\hbar$. 


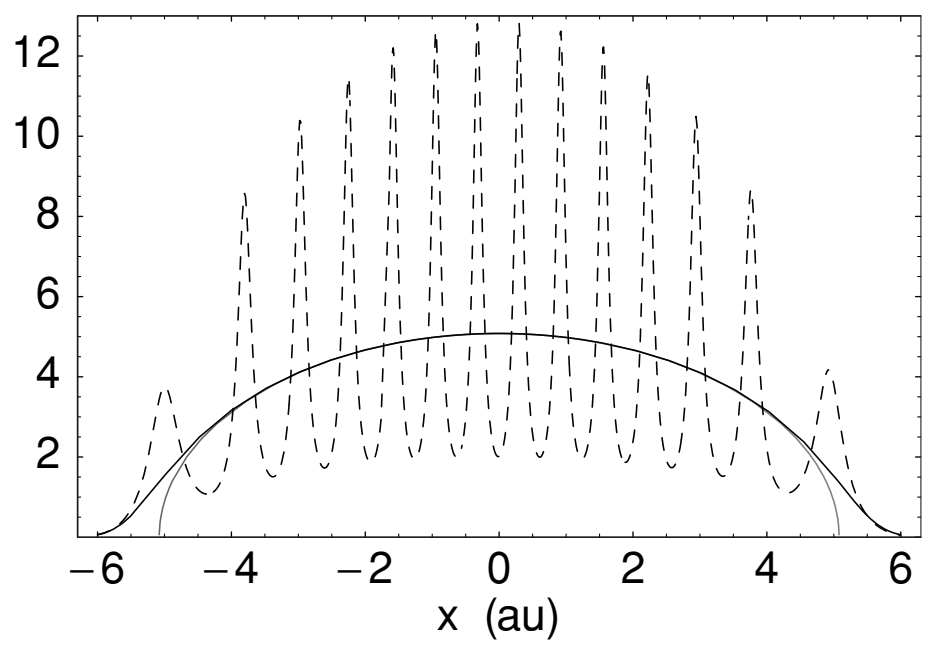

Figure 2. The positive branch of the classical canonical momentum $p(x)$ for a harmonic oscillator $\left(\omega=1\right.$, atomic units) is plotted (lower solid line) versus the 'quantal momentum' $\partial_{x} \phi(x, c) i$ ) for an arbitray value of $c$ (dashed line) and $i$ ) for the non-oscillating value $c=c_{0}$ (upper solid line).

obtained from these classical quantities. Semi-classical quantization must take into account the singularities at the turning points, from which it follows that $E_{0}=\hbar \omega(n+1 / 2)$, with $n$ being an integer.

Quantum mechanically, the eigenvalues are given by $\xi(n)=\hbar \omega(n+1 / 2)$ when $n$ is an integer that counts the zeros of the eigenfunction, but this relation can be extended for any real value of $n$, as discussed in section 2.3.4. We then write $\xi^{-1}$ as $n(E)=E / \hbar \omega-1 / 2$. The derivative of the exact (quantum) phase is given by equations (7) and (8) as

$\partial_{x} \phi(x, c)=\frac{m \omega}{\hbar}\left[\left(\frac{1}{2 I}+2 I c^{2}\right) u_{1}^{2}(x)+\frac{2 I}{W^{2}} u_{2}^{2}(x)-\frac{4 I c}{W} u_{1}(x) u_{2}(x)\right]^{-2}$.

Figure 2 compares the classical conjugate momentum as given by equation (43) with two exact phases $\hbar \partial_{x} \phi(x, c)$ obtained from equation (45) by using numerical solutions of the Schrödinger equation, $u_{1}$ and $u_{2}$, with respective vanishing boundary conditions at $x=-\infty$ and $x=+\infty$. One of the curves is for an arbitrary value of $c$, the other corresponds to $c=c_{0}$. It may be seen that even for a moderate excitation $(n \approx 12)$, the non-oscillating solution with $c=c_{0}$ can barely be distinguished from the classical momentum at the same energy, except near the turning points (the reason is that $\langle S ; x\rangle$ is negligible, hence the exact quantum phase, solution of equation (6) tends to the semi-classical quantum phase $\tilde{\phi}$ of equation (28), which is simply $S(x)$ when $\left.c=c_{0}\right)$.

The analogue of the canonical action $J$ appears as the line integral (41), which generally depends on $c$ except when $n$ is an integer in which case $\oint\left[\hbar \partial_{x} \phi(x, c)\right] \mathrm{d} x=2 \pi \hbar n$. Thus for the quantized energies, the quantal line integral differs from $J$ by the Maslov index. However, if $c=c_{0}$, we have for any $E$ [equation (42)]

$$
\oint\left[\hbar \partial_{x} \phi\left(x, c_{0}\right)\right] \mathrm{d} x=2 \pi E / \omega-\hbar / 2
$$

which is the classical result with an action correction coming from the Maslov index. Note that taking the energy derivative of equation (41) crucially depends, for any energy $E$ (including the eigenvalues), on the energy dependence $c(E)$. Only the energy dependence given by the 
relation (23), which yields equation (46) in this case, renders the usual relation for the period, and more generally follows the classical time parameterization for conservative systems in the Hamilton-Jacobi theory. Thus, the parameter $c$, which appears free within quantum mechanics and has no classical counterpart, must be constrained if the usual classical relations for the oscillator are to be extended to quantum amplitude and phase functions.

The usual interpretation of the invariant within Ermakov systems hinges on the use of an original Hamiltonian or Lagrangian, from which the Ermakov equations are derived. The invariant is then associated, by means of Noether's theorem, with the conserved quantity of an auxiliary motion $[5,6]$. Such an interpretation is of course not available here, where the Ermakov equations are used in a quantum-mechanical context. The invariant $I$ is employed in this context only to define the normalization, through equation (12). However, for the choice $c=c_{0}$, a further interpretative step may be taken, since then the term on the right-hand side in equations (12) or (22) gives

$$
\frac{\hbar}{2 m} I \partial_{E} \oint\left[\hbar \partial_{x} \phi\left(x, c_{0}\right)\right] \mathrm{d} x .
$$

For the harmonic oscillator, we have by equation (46) and by adopting unity normalization

$$
I=\frac{m \omega}{\hbar \pi} \text {. }
$$

For other systems, $I$ usually depends on $n(E)$ except if the wavefunctions are energynormalized. Elementary manipulations yield the more general form

$$
I=2 \pi\left[\int \lambda(x, E) \mathrm{d} x\right]^{-1}
$$

where $\lambda(x, E)$ is the local de Broglie wavelength and the range of integration is restricted to the classical domain between the turning points.

\section{Conclusion}

Previous interest in amplitude-phase methods led us to investigate in this work the oscillatory properties of the non-linear equation of uncoupled Ermakov systems. It was shown that non-oscillating amplitude-phase functions in the space and energy variables have a particular feature in the semi-classical limit: they yield classical quantities. We have seen that although standard quantum-mechanical quantities, such as the wavefunctions or the eigenvalues, are insensitive to the value of the parameter $c$ and its energy dependence, there is a unique value of $c$ which appears as connecting quantum amplitude and phase functions to their classical counterpart. Only in this case can 'quantum characteristics' $\left(x, \partial_{x} \phi\right)$ having a sense, and collapsing to $\left(x, \partial_{x} S\right)$ when $\hbar \rightarrow 0$ be defined. We insist again that from the point of view of quantum mechanics, even in the semi-classical limit, this need not be the case: any amplitude and phase functions obeying equation (33) will yield correct semi-classical wavefunctions. A full study of these aspects on specific physical systems will be given elsewhere. Further links with current work on Ermakov systems may lead to a better appreciation, as well as to an extension, of the formalism.

\section{Acknowledgments}

I thank T Monteiro (UCL) for useful discussions on semi-classical physics. Financial support from the European Commission's IHP-MCIF Programme is acknowledged. 


\section{References}

[1] Haas F and Goedert J 1999 J. Phys. A 322835

[2] Schief W K, Rogers C and Bassom A P 1996 J. Phys. A 29903

[3] Kaushal R S, Parashar D, Gupta S and Mishra S C 1997 Ann. Phys. (N.Y.) 259233

[4] Ray J R and Reid J L 1979 J. Math. Phys. 202054

[5] Reid J L and Ray J R 1980 J. Math. Phys. 211583

[6] Lewis H R, Leach P G L, Bouquet S and Feix M R 1992 J. Math. Phys. 33591

[7] Simic S S 2000 J. Phys. A 335435

[8] Matzkin A 2001 Phys. Rev. A 63012103

[9] Levitan B M and Sargsjan I S 1991 Sturm-Liouville and Dirac Operators (Dordrecht: Kluwer)

[10] Fedoriuk M V 1993 Asymptotic Analysis (New York: Springer)

[11] Lewis H R 1968 J. Math. Phys. 91976

[12] Kruskal M 1962 J. Math. Phys. 3806

[13] Greene C H, Rau A R P and Fano U 1982 Phys. Rev. A 262441

[14] Rodberg L S and Thaler R M 1967 Introduction to the Quantum Theory of Scattering (New York: Academic)

[15] Seaton M J 1983 Rep. Prog. Phys. 46167

[16] Matzkin A 1999 Phys. Rev. A 592043

[17] Campos D, Urbina J D and Viviescas C 2000 J. Phys. A 336129

[18] Leacock R A and Padgett M J 1983 Phys. Rev. Lett. 503

[19] Voros A 2000 J. Phys. A 337423

[20] Goldstein H 1980 Classical Mechanics (Reading, MA: Addison-Wesley)

[21] Percival I C 1977 Adv. Chem. Phys. 361 